\title{
Spatiotemporal vortex solitons in hexagonal arrays of waveguides
}

\author{
Hervé Leblond, ${ }^{1}$ Boris A. Malomed, ${ }^{2,3}$ and Dumitru Mihalache ${ }^{4,5,1}$ \\ ${ }^{1}$ Laboratoire de Photonique d'Angers, EA 4464, Université d'Angers, 2 Bd Lavoisier, F-49000 Angers, France \\ ${ }^{2}$ Department of Physical Electronics, School of Electrical Engineering, Faculty of Engineering, Tel Aviv \\ University, Tel Aviv IS-69978, Israel \\ ${ }^{3}$ ICFO-Institut de Ciencies Fotoniques, Mediterranean Technology Park, E-08860 Castelldefels (Barcelona), Spain \\ ${ }^{4}$ Horia Hulubei National Institute for Physics and Nuclear Engineering (IFIN-HH), 407 Atomistilor, \\ Magurele-Bucharest, R-077125, Romania \\ ${ }^{5}$ Academy of Romanian Scientists, 54 Splaiul Independentei, R-Bucharest 050094, Romania
}

(Received 9 May 2011; published 20 June 2011)

\begin{abstract}
By means of a systematic numerical analysis, we demonstrate that hexagonal lattices of parallel linearly coupled waveguides, with the intrinsic cubic self-focusing nonlinearity, give rise to three species of stable semidiscrete complexes (which are continuous in the longitudinal direction) with embedded vorticity $S$ : triangular modes with $S=1$, hexagonal ones with $S=2$, both centered around an empty central core, and compact triangles with $S=1$, which do not not include the empty site. Collisions between stable triangular vortices are studied too. These waveguiding lattices can be realized in optics and Bose-Einstein condensate.
\end{abstract}

DOI: 10.1103/PhysRevA.83.063825

PACS number(s): 42.65.Tg, 42.81.Dp, 03.75.Lm, 05.45.Yv

\section{INTRODUCTION}

Lattice solitons are a topic of great interest to ongoing studies of nonlinear dynamics in photonic media and BoseEinstein condensates (BECs) [1]. These localized modes are produced by the interplay of the intrinsic nonlinearity of the medium with an effective periodic potential induced in it by permanent or virtual lattice patterns. In fact, the lattice may itself be a nonlinear structure if it is induced by a spatially periodic modulation of the local nonlinearity [2]. In the limit of a deep periodic potential, the fundamental models of lattice media reduce to various versions of the discrete nonlinear Schrödinger (DNLS) equation [3]. Realization of the onedimensional (1D) DNLS model in arrayed optical waveguides was originally proposed in Ref. [4]. The same model was later applied to BECs loaded into deep optical-lattice potentials [5] (see Ref. [6] for a brief review). A physical realization of the DNLS model is also possible in the form of lattices of microcavities which serve as traps for polaritons [7].

Lattice solitons take the form of discrete solitons in terms of the DNLS equations, which correspond to quasidiscrete solitons in the respective experimental settings. Such solitons were created in a set of semiconductor waveguides built on top of a slab substrate [8], and also in arrays of optical fibers [9]. In addition to using permanent photonic structures, quasidiscrete solitons were also made in virtual waveguiding arrays, using the versatile technique of inducing interference lattices in photorefractive crystals [10]. The latter method was used to create the first examples of two-dimensional (2D) quasidiscrete fundamental solitons [11], which was followed by the making of vortex solitons [12], i.e., localized lattice excitations with embedded vorticity that were predicted in Ref. [13]. Another significant contribution to this area was the creation of 2D solitons in a bundle of fiberlike waveguides written in bulk silica [14]. Such arrays and bundles are created by means of tightly focused femtosecond laser pulses [15].

Following the analysis of the fundamental localized discrete vortices with topological charge $S=1$ [13], their higher-order counterparts, with $S>1$, and multipole discrete solitons, such as quadrupoles, were predicted in Refs. [16,17]. Many other objects were studied in this area, including supervortices (circular chains of compact vortices with an imprinted overall topological charge, which is independent of the vorticity of the individual eddies [17]), necklace-shaped patterns [18], discrete solitons in hexagonal and honeycomb lattices [19,20], composite semidiscrete spatial solitons in arrays of waveguides with quadratic and cubic nonlinearities [21], quasidiscrete topological solitons in photonic-crystal fibers [22], etc. Nonstationary soliton effects were studied too. These include the mobility of discrete solitons $[23,24]$, collisions between traveling ones [24,25], and the onset of the spatiotemporal collapse in self-focusing arrayed waveguides [26].

Most works on lattice solitons dealt with the spatial-domain settings. In particular, optically induced lattices in photorefractive crystals do not make it possible to observe the evolution in the temporal domain because of a very large response time in these materials. However, the spatiotemporal dynamics can be realized in waveguiding arrays written in bulk silica [15], where the spatially localized quasidiscrete patterns in the transverse plane can be combined with the temporal selftrapping in the longitudinal direction. Recently, the creation of the corresponding quasidiscrete "light bullets" was reported in this system [27] (for a review of spatiotemporal solitons in nonlinear optics and BEC, see Ref. [28]). Previously, a number of manifestations of the spatiotemporal self-trapping in similar settings were studied theoretically, including the related modulational instability [29], formation of "bullets" in fiber arrays [30] and photonic wires [31], and self-compression [32] and steering [33] of pulsed beams. Continuing the work in this direction, semidiscrete spatiotemporal surface solitons were introduced as surface modes in semi-infinite waveguide arrays [34] and in a system with an interface between different arrays [35]. Also analyzed were spatiotemporal solitons in waveguide arrays with the quadratic nonlinearity [36].

Once stable fundamental spatiotemporal soliton complexes in bundled arrays of waveguides are available, it is natural to seek for vortex solitons in the same setting. A systematic 
analysis of spatiotemporal vortices and quadrupoles in the model based on the square lattice of discrete waveguides was reported in Ref. [37]. A vast stability area was found for the solitary vortices with $S=1$ and quadrupoles, which are built as rhombuses, alias on-site-centered modes, with respect to the underlying lattice (the rhombus is built as a set of four "bright" cores, with a nearly "dark" one at the center). The stability region is much smaller for the off-site-centered modes of the "square" type, without an empty pivotal site in the middle (the reduced stability domain of square-shaped vortices and quadrupoles, in comparison with their rhombic counterparts, in a generic feature of topological solitons in lattice media [38]). All the spatiotemporal vortex solitons with $S=2$ were found to be unstable in the same model. Further, collisions between stable vortices and quadrupoles (with identical or opposite topological charges), propagating along the bundle in opposite directions, were analyzed in Ref. [39]. Four different outcomes of the collisions were identified: rebound of slowly moving solitary vortices, fusion, splitting, and quasielastic interactions between fast ones.

Hexagonal lattices may be created by means of the same techniques which were used for building the square-shaped structures. On the other hand, the change of the underlying geometry may essentially alter fundamental properties of topological lattice solitons $[3,19]$. In particular, it was predicted theoretically and confirmed in an experiment that spatial solitons in the form of double vortices (with $S=2$ ) in hexagonal lattices may be stable, while their unitary counterparts (with $S=1$ ) are unstable.

The objective of the present work is to study spatiotemporal vortex solitons in hexagonal lattices of discrete waveguides. The model is formulated in Sec. II, and at the end of it we also briefly consider fundamental solitons, driven by a temporally self-trapped pulse in a single waveguiding core. In Sec. III we demonstrate, also in a brief form, that a straightforward input in the form of a hexagon-shaped spatiotemporal vortex with $S=1$ always leads to a decay. Nevertheless, three different species of stable spatiotemporal complexes with the embedded vorticity are revealed by a systematic numerical analysis. In Sec. IV we demonstrate that a spatiotemporal input of a triangular shape generates self-trapped vortices in the form of triangles with an empty core in the middle. Further, in Sec. V it is shown that a hexagonally shaped input with $S=2$ produces stable spatiotemporal hexagons with the same (double) topological charge. Finally, a modified (shifted) input ansatz gives rise to stable densely packed triangular vortices with $S=1$, without an empty central core, as shown in Sec. VI. In addition to the study of these species of spatiotemporal vortex solitons, in Sec. VII collisions between counterpropagating triangular ones are studied. The paper is concluded by Sec. VIII.

\section{THE MODEL AND FUNDAMENTAL SOLITONS}

We consider the hexagonal array of nonlinear waveguides with cells in the transverse lattice numbered as shown in Fig. 1. The transmission of waves in the array is described by the following system of coupled NLS equations, written

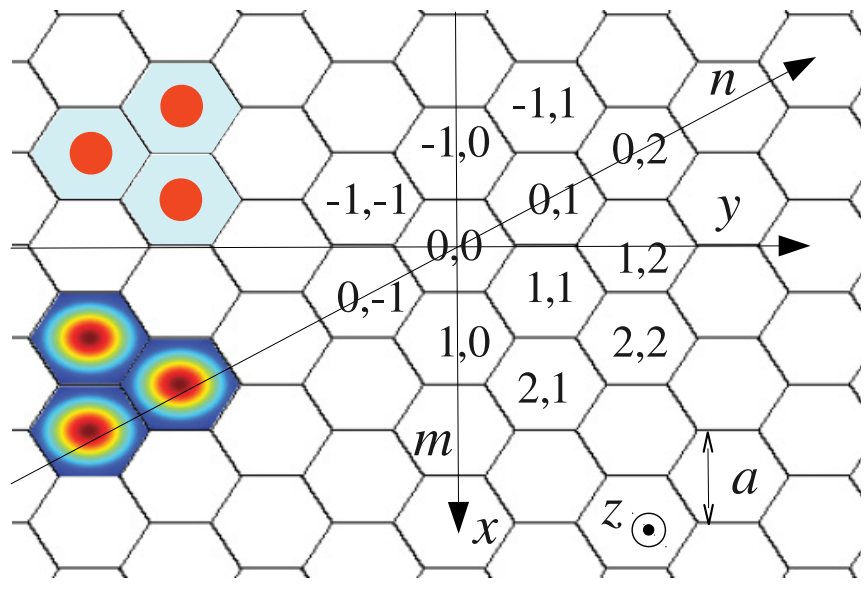

FIG. 1. (Color online) The setup and notation: We consider the hexagonal array of cylindrical waveguides, as shown in the upper left corner. The transverse distribution of the light intensity of the propagating waves in the guiding cores is displayed symbolically in the bottom left corner. Each core is assumed to be a singlemode waveguide, represented by wave function $u_{m, n}$, with discrete coordinates $(m, n)$ defined as shown in the figure. The map of integers $m, n$ into the Cartesian coordinates in the transverse plane, $(x, y)$, is performed as per Eq. (5), $a$ being the width of the hexagonal cell.

in the scaled form, similar to that used in many earlier works [27,29-37]:

$$
\begin{aligned}
i \partial_{z} u_{m, n}+ & {\left[u_{m-1, n-1}+u_{m-1, n}+u_{m, n-1}+u_{m, n+1}\right.} \\
& \left.+u_{m+1, n}+u_{m+1, n+1}-(6+\mu) u_{m, n}\right] \\
& +(1 / 2) \partial_{t}^{2} u_{m, n}+u_{m, n}\left|u_{m, n}\right|^{2}=0 .
\end{aligned}
$$

In terms of the optical setting, $z$ and $t$ are, respectively, the propagation distance and reduced time, assuming that each guiding core features the anomalous chromatic dispersion and cubic self-focusing, while $-\mu$ is the propagation constant of the localized solution to be sought for. In terms of the corresponding BEC model, Eqs. (1) is a system of coupled discrete Gross-Pitaevskii equations [40], with $z$ and $t$ playing the roles of the scaled time and axial coordinate, respectively, while $\mu$ is the chemical potential.

Simulations of Eq. (1) were carried out in the Fourier domain with the help of the standard fourth-order Runge-Kutta scheme, the nonlinear term being evaluated by means of the combination of inverse and direct fast Fourier transforms at each substep of the scheme. We used an $11 \times 12$ matrix in the plane of $(m, n), 512$ points for variable $t$ in the computation window of width $\Delta t=20$, and the step size in the propagation direction $d z=5 \times 10^{-4}$. Use of the Fourier transform implies periodic boundary conditions in $t$, which make sense if a characteristic temporal size of the localized objects will be essentially smaller than $\Delta t=20$. As concerns the boundary conditions for the discrete coordinates $m$ and $n$, the values of $u_{m, n}$ corresponding to the coordinates which fall outside of the computation box are replaced by zeros.

Before proceeding to the search for complex spatiotemporal vortical patterns, it makes sense to test the propagation of fundamental solitons, which are carried, essentially, by 


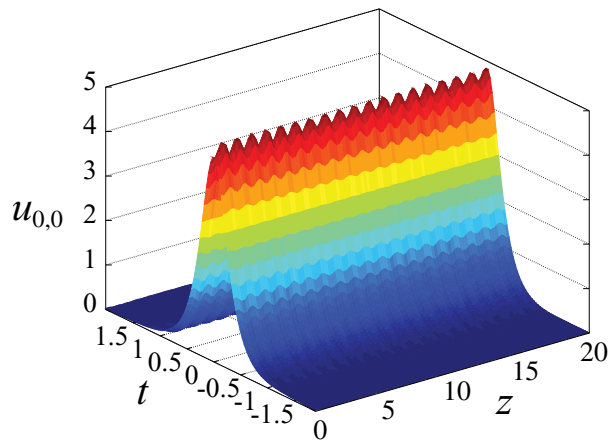

(a)

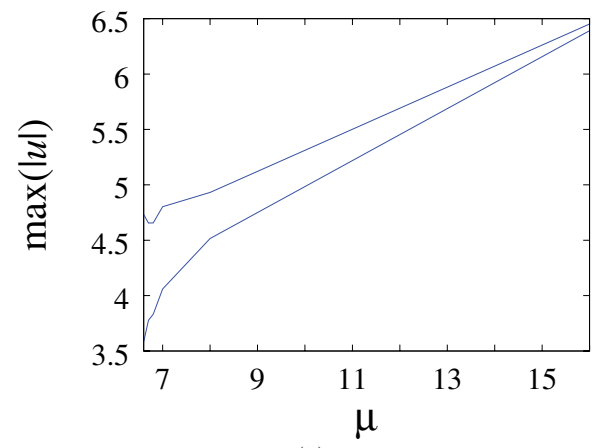

(b)

FIG. 2. (Color online) (a) Oscillations of the fundamental soliton generated by input (2), (3) with $\mu=8$. (b) The upper and lower curves illustrate the oscillations by showing, respectively, the largest and smallest values of the soliton's amplitude, i.e., $\max _{z}\left[\max _{t}(|u|)\right]$ and $\min _{z}\left[\max _{t}(|u|)\right]$, as functions of the propagation constant, $\mu$.

a temporal pulse in a single core. For this purpose, the simulations were initiated with obvious initial conditions,

$$
\begin{gathered}
u_{0,0}(z=0)=\eta \operatorname{sech}(\eta t), \\
\eta=\sqrt{2(6+\mu)},
\end{gathered}
$$

setting $u_{m, n}(z=0)=0$ at $|m|+|n| \neq 0$. The simulations were run in the interval $1 \leqslant \mu \leqslant 16$ of values of the propagation constant.

It has been concluded that input (2) decays, under the action of the lattice diffraction, at $\mu<6.6$, and a stable fundamental soliton, concentrated in the central core, is formed at $\mu \geqslant 6.6$. The temporal pulse which lies at the core of the so-created fundamental soliton is not quite stationary but rather features regular pulsations, as shown in Fig. 2.

One may surmise that the oscillations of the fundamental soliton could be a result of its interaction with the radiation background, which was generated by the input field in the course of self-trapping into the fundamental soliton. To check this possibility, the background around the soliton was explicitly removed at a particular step of the simulations. Nevertheless, the oscillations remain virtually unaffected by the "cleaning," i.e., they seem to be a genuine feature of the dynamics of the soliton, possibly representing its intrinsic mode.

\section{THE HEXAGONAL INPUT: A TRANSITION TO INSTABILITY}

First we attempted to create hexagonal vortical modes with $S=1$, which seems a natural approach to the system based on a hexagonal lattice. To this end we used the following input, based on an ansatz factorized in the longitudinal (temporal) and transverse (spatial) directions (cf. Ref. [41]):

$$
\begin{aligned}
u_{m, n}= & \frac{\left(x_{m, n}+i y_{m, n}\right)}{a} \\
& \times \exp \left[-\alpha\left(\sqrt{x_{m, n}^{2}+y_{m, n}^{2}}-a\right)\right] \eta \operatorname{sech}(\eta t),
\end{aligned}
$$

with $\eta$ taken as per Eq. (3), and

$$
\begin{gathered}
x_{m, n} \equiv a\left(m-\frac{n}{2}\right), y_{m, n} \equiv \frac{\sqrt{3}}{2} a n, \\
\alpha=\ln [2(6+\mu)],
\end{gathered}
$$

$a$ being the width of the hexagonal cell (see Fig. 1). The model's scale is fixed by setting $a \equiv 1$. Factor $\left(x_{m, n}+i y_{m, n}\right)$ in Eq. (4) obviously corresponds to vorticity $S=1$, and the exponential factor with $\alpha$, taken as per Eq. (6), is determined as in the $2 \mathrm{D}$ spatial soliton with propagation constant $-\mu$. The choice of $\eta$ as per Eq. (3) implies that, simultaneously, the wave field in the factorized ansatz is localized in the longitudinal direction, in each core, as in the temporal soliton corresponding to the same propagation constant, cf. the structure of the fundamental solitons considered above. The phase and energy structure of ansatz (4) is illustrated in a schematic form by Fig. 3.

Direct simulations of Eq. (1) with this input have been run in a broad range of values of the propagation constant, $7<$ $\mu<500$. Nonetheless, stable spatiotemporal vortices with the hexagonal structure and topological charge $S=1$ have never emerged. In fact, the evolution of the input organized as the ansatz of this type never leads to formation of any stable pattern. In the interval of $7 \leqslant \mu \leqslant 13$, the system makes an attempt to generate a robust pattern of a triangular shape, as shown in Fig. 4: at three sites belonging to the original hexagon the field quickly decays, while at three others it survives, for a while. However, the largest amplitude of the temporarily emerging triangular set is $\simeq 8$ (it is attained at $\mu=13$ ), while

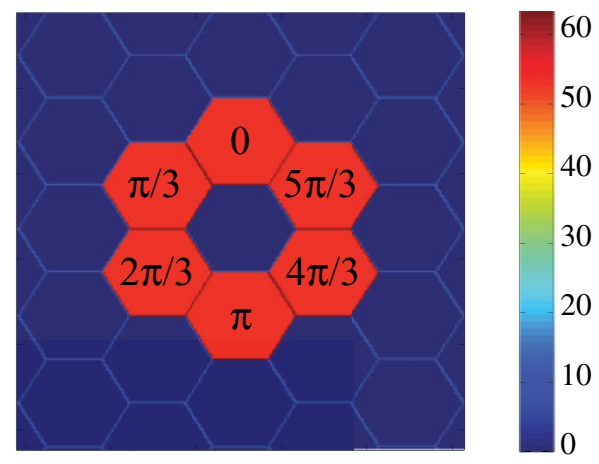

FIG. 3. (Color online) The phase and energy $\left(\int_{-\infty}^{+\infty}\left|u_{m, n}\right|^{2} d t\right)$ patterns corresponding to input ansatz (4) which generates an (unstable) hexagonal vortex with $\mu=350$. 

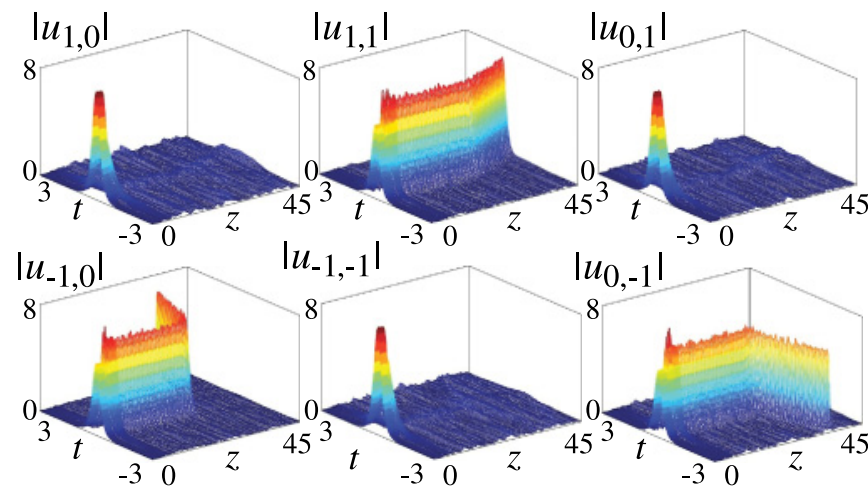

$\left|u_{-1,-1}\right|$
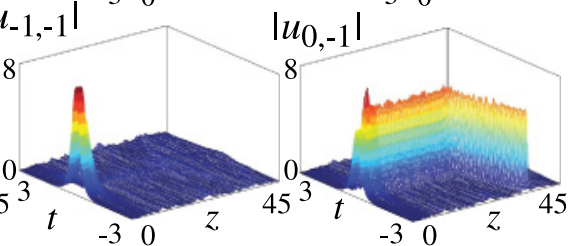

FIG. 4. (Color online) The evolution of the six main components of the hexagon with initial propagation constant $\mu=9$. In this case, the simulations demonstrate the decay of the hexagon into a transient triangle, which is followed by a longitudinal instability (splitting).

triangular vortices may be stable for amplitudes above a threshold value of the amplitude which is $\simeq 18$ (see below). Therefore the triangles developing from the unstable hexagons are also subject to an instability, eventually splitting into uncorrelated single-core excitations which separate in the longitudinal direction (see Fig. 4).

Further, in the interval of $13<\mu \leqslant 70$, the instability splits the original hexagon into a set of separating single-core excitations, the number of which varies randomly between 2 and 6 (not shown here in detail). In an adjacent interval, $100 \leqslant$ $\mu<200$, the instability-development scenario is similar but faster so that the formation of a transient triangular structure cannot be identified.

At largest values of the propagation constant, $200 \leqslant$ $\mu \leqslant 500$, the instability-development scenario is different. The six sites forming the hexagon keep their positions and amplitudes for a while but loose the mutual phase coherence. Then instabilities of amplitudes and positions set in, but they manifest themselves on a much longer scale of the propagation distance, with $z$ ranging from 10 to a few hundreds, instead of $z \sim 1$ at small $\mu$ (cf. Fig. 4). The separation between excitations in individual cores grows very slowly too, in comparison with the quick split of the transient triangle observed in Fig. 4 .

\section{THE GENERATION OF STABLE TRIANGULAR VORTICES}

The next step is an attempt to generate a triangular vortical structure, which is suggested by the emergence of a transient one in the course of the evolution of the unstable hexagon (Fig. 4). For this purpose we used the same input as defined by Eqs. (4)-(6) but with three main peaks suppressed, which was done by replacing the fields at the corresponding sites by those from adjacent sites in the outer layer: $u_{-1,0} \rightarrow u_{-2,0}, u_{1,1} \rightarrow$ $u_{2,2}, u_{0,-1} \rightarrow u_{0,-2}$, as shown in Fig. 5. The so-constructed triangular ansatz keeps the vorticity of the original hexagon, $S=1$.

The evolution of this input was simulated in a broad range of values of the propagation constant, $4 \leqslant \mu \leqslant 450$. At small values, $\mu \leqslant 6$, the three main peaks forming the triangle merge into a single-core fundamental soliton, which may be localized

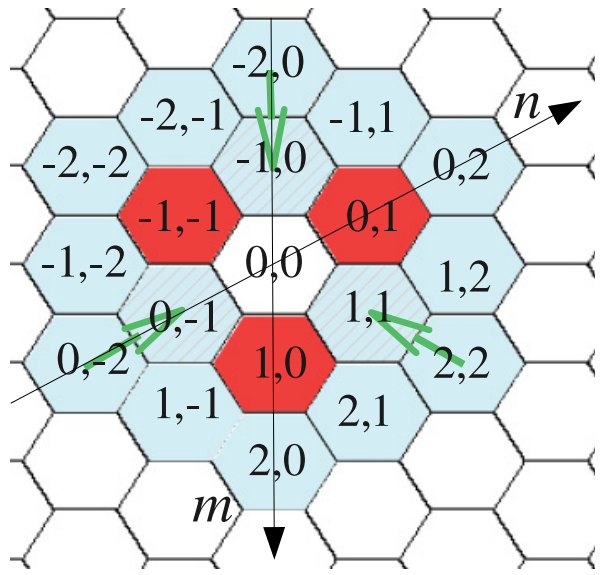

FIG. 5. (Color online) The reduction of the hexagonal input to the triangular-vortex one: three of the six main peaks are replaced by fields taken from the surrounding layer, as shown by arrows. (The replacement makes the amplitudes at the corresponding sites much smaller but does not alter their phases.)

at the central site or at any one site belonging to the original triangle. Thus stable vortices do not emerge in this case. For $7 \leqslant \mu \leqslant 181$, the triangle is destroyed by an instability which splits it into separating uncorrelated single-core excitations (the instability develops faster with the increase of $\mu$ ).

Finally, the same input generates stable triangular vortices at $\mu \geqslant 182$, an example of which is shown in Fig. 6. The stability was verified by direct simulations for long propagation distances, e.g., $z=986$ for $\mu=182$. The temporal pulses in the cores which represent vertices of the triangle remain well phase-locked, keeping the phase circulation of $2 \pi$, which corresponds to vorticity $S=1$. The excitations at secondary sites (between the vertices) are phase-locked to the primary ones, but featuring some oscillations. The oscillations enhance with $\mu$, but the overall vortical phase pattern always persists. On the other hand, the amplitudes of excitations at the secondary sites feature fast irregular oscillations, which also become stronger at larger $\mu$ (variations of these amplitudes by a factor of $\sim 2$ are observed already at the stability threshold, $\mu=182$ ). These amplitude oscillations are coupled to small variations of amplitudes at the primary sites, as shown in Fig. 7. It has been checked that the oscillations were not induced by reflection of small-amplitude radiation waves from edges of the integration domain (absorbers installed at the edges do not suppress the oscillations).

Figure 8 displays the total energy, $E=\sum_{m, n} \int_{-\infty}^{+\infty}$ $\left|u_{m, n}(t)\right|^{2} d t$, and amplitude of the triangular vortices, both stable and unstable ones, as functions of the effective propagation constant $\mu+\delta \mu$, where the contribution $\delta \mu$ from oscillations of the fields is computed as follows. For each vertex of the triangle $(m, n)$, peak time $t_{m, n}$ is defined such that $\left|u_{m, n}\left(t_{m, n}\right)\right|=\max _{t}\left|u_{m, n}(t)\right|$ and the corresponding phase, $\phi_{m, n}\left(t_{m, n}\right)$, is identified. Next we compute

$$
\delta \mu=\left\langle\frac{d}{d z} \phi_{m, n}\right\rangle,
$$

where the average is taken over the three vertices of the triangle (or six ones for stable hexagonal vortices with $S=2$, see the 


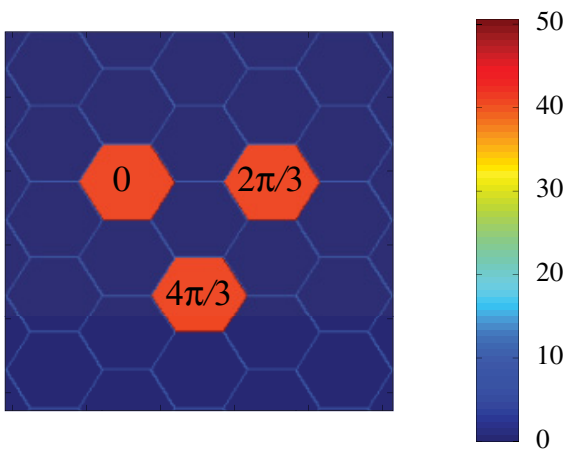

(a)

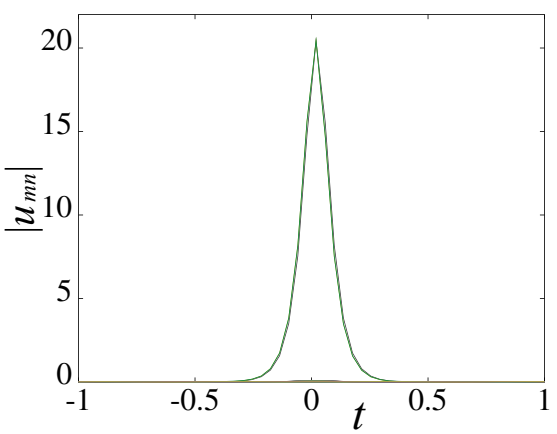

(b)

FIG. 6. (Color online) (a) The transverse energy $\left(\int_{-\infty}^{+\infty}\left|u_{m, n}\right|^{2} d t\right)$ and phase profile of the stable triangular vortex generated by the input with $\mu=200$. (b) The longitudinal (temporal) profile of excitations in the cores representing the vertices of the triangle.

next section), and for the stable triangular modes, over $z$. For unstable triangles, the latter average was taken over a short interval $\Delta z$, within which the pattern was not disturbed by the instability. With regard to the amplitude shown in Fig. 8, it was defined as $\left|u_{m, n}\left(t_{m, n}\right)\right|$, averaged over $z$ and over the three vertices, to smooth effects of small persistent oscillations of the local amplitudes. A small gap between the unstable and stable portions of the amplitude plot in Fig. (7) is due to the
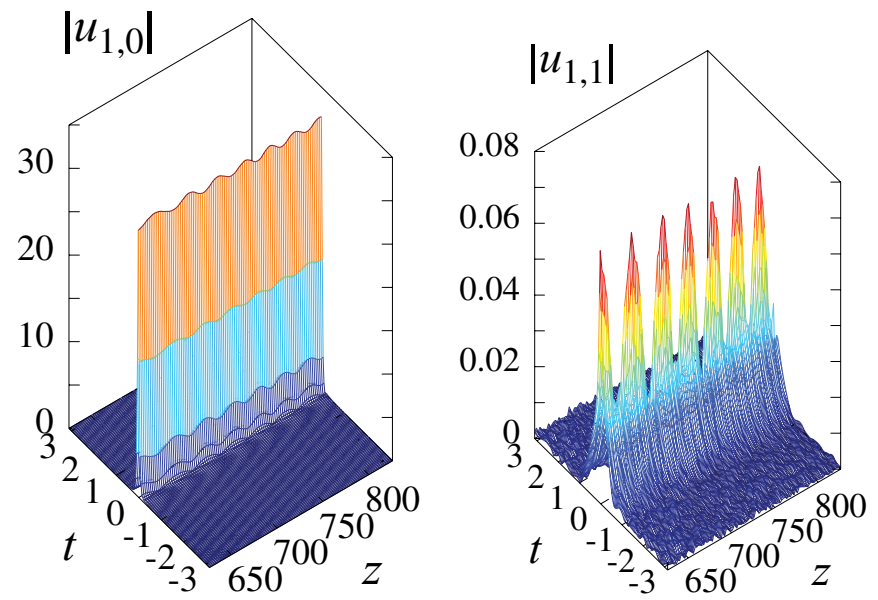

FIG. 7. (Color online) The evolution of the longitudinal (temporal) profiles of one primary and one secondary component of a stable triangular spatiotemporal vortex, generated by the triangular input with $\mu=450$.
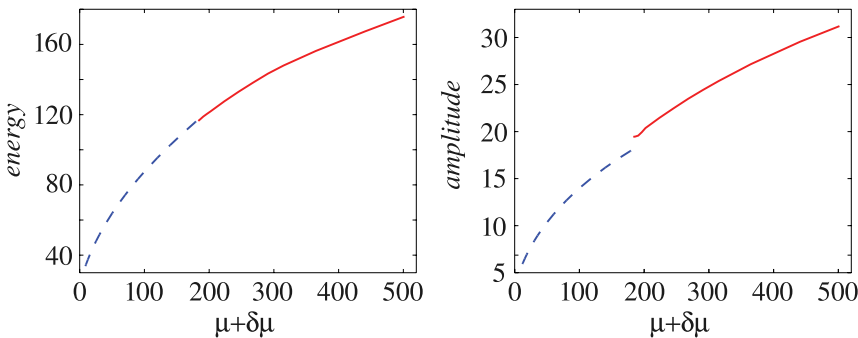

FIG. 8. (Color online) The amplitude and energy of the stable triangular vortex vs the effective propagation constant $\mu+\delta \mu$ [see Eq. (7)]. Blue (dashed) and red (solid) segments designate unstable and stable solution families, respectively.

difference in wave-number shift (7), as computed for the stable and unstable solutions.

\section{HEXAGONAL VORTICES WITH THE DOUBLE TOPOLOGICAL CHARGE}

As said above, the hexagonal input based on Eqs. (4)-(6) could not produce any stable pattern. However, the same initial ansatz, but with inverse signs of three of its main peakssay $u_{-1,0}, u_{1,1}, u_{0,-1}$ - can give rise to stable hexagonal spatiotemporal patterns carrying vorticity $S=-2$ (if the topological charge of the original ansatz is defined as $S=+1$ ), see an example of the stable mode in Fig. 9. Note that, in this case, no changes were made to the hexagonal input at sites in outer layers.

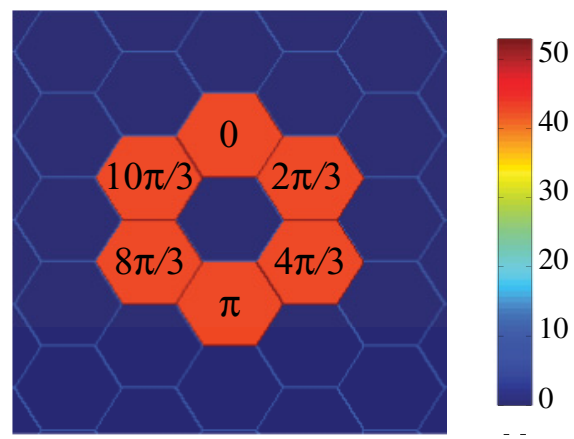

(a)

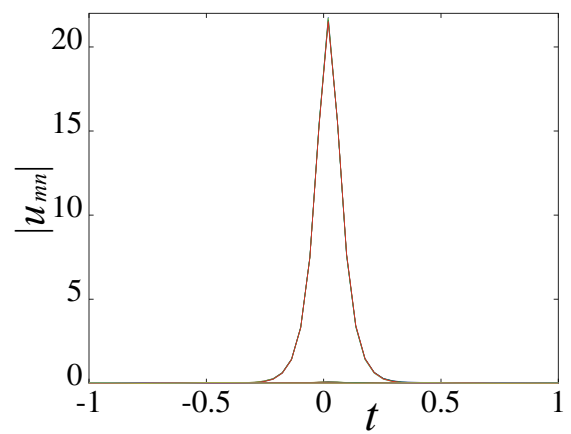

(b)

FIG. 9. (Color online) The same as in Fig. 6 but for a stable hexagonal vortex with topological charge $S=2$, generated by the modified input with $\mu=223$. 

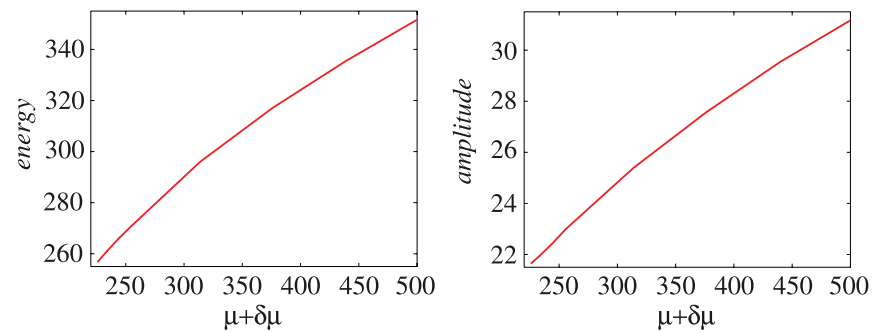

FIG. 10. (Color online) The same as in Fig. 8 but for the hexagon-shaped vortices with the double topological charge. Only stable modes are presented in these plots.

Simulations with this input were also run in a broad range of values of the propagation constant, $5 \leqslant \mu \leqslant 450$. At $\mu<223$ the seeded pattern is subject to various instabilities: spread out due to the lattice diffraction and temporal dispersion, or splitting into separating excitations, or, sometimes, merging into one or two single-core fundamental solitons. In particular, in the interval $8 \leqslant \mu \leqslant 10$ the pattern forms a transient triangular structure, which eventually splits, and in a broad interval of $20 \leqslant \mu \leqslant 222$ the initial hexagon fissions into two triangles, which also turn out to be unstable, essentially, because the amplitudes of the triangular patterns fall below the stability threshold.

Stable double $(S=2)$ hexagonal vortices emerge at $\mu \geqslant 223$, whose shape is illustrated by Fig. 9. The stability of these vortices was verified in very long simulations. With the further increase of $\mu$, an instability island was revealed around $\mu=300$. In that case, the six temporal pulses remain locked to their positions, but the phase structure is lost at $z \gtrsim 500$. Nevertheless, the hexagonal vortices recover their integrity at still larger $\mu$. It is possible that other narrow intervals of the instability may be found inside the stability region.

Figure 10 presents the energy and amplitude of the double vortices as functions of the effective wave number, similar to Fig. 8 for the triangular vortices with $S=1$. However, only the stable family of the hexagonal vortices is shown here, as we were not able to measure characteristics of unstable ones at $\mu<223$. In fact, the simulations produce no evidence that such unstable modes exist.

\section{COMPACT TRIANGULAR VORTICES}

Still another type of stable spatiotemporal pattern can be produced by the input taken as per Eqs. (4)-(6), but centered at an edge of the original hexagon, i.e., with $m$ and $n$ replaced by $m-2 / 3$ and $n-1 / 3$, respectively. In this case the results were collected for $5 \leqslant \mu \leqslant 220$, and as shown in Fig. 11, the stable structure takes the form of a vortex with $S=1$, shaped as a densely packed triangle, without an empty site in the center (cf. Fig. 6). This structure is stable for $\mu \geqslant 11$. It is relevant to stress that this stabilization threshold is more than an order of magnitude lower than its counterparts for the triangular and hexagonal spatiotemporal vortices reported in the previous sections (recall those thresholds were $\mu_{\text {triangle }}=$ 182 and $\mu_{\text {hexagon }}=223$, respectively).

In fact, direct simulations initiated by the above-mentioned shifted input ansatz generate the compact triangle which seems

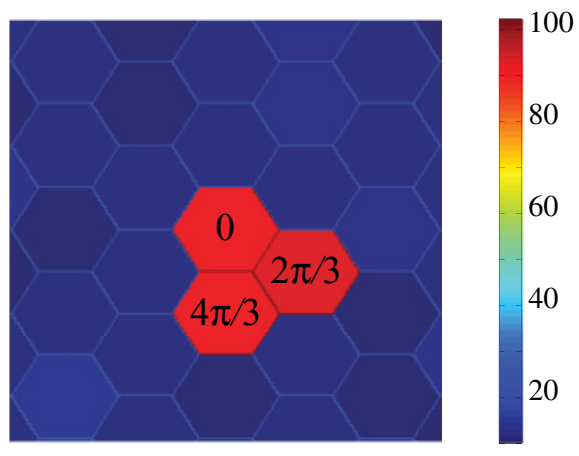

(a)

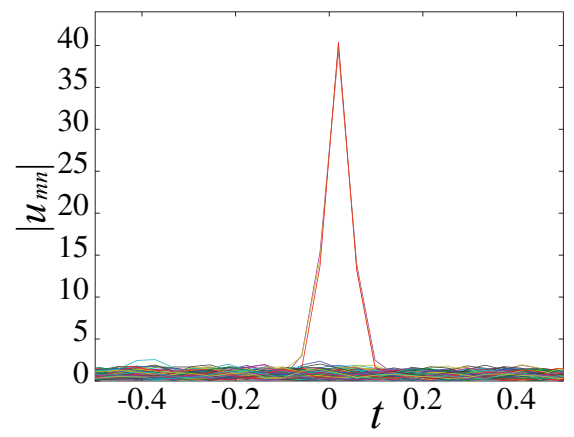

(b)

FIG. 11. (Color online) The same as in Fig. 6 but for a stable compact triangular vortex with topological charge $S=1$, generated by the shifted input with $\mu=15$.

"noisy." The noise can be removed by means of the "temporal filtering," setting the field equal to zero outside of the main pulse in each core and running the additional propagation over $\Delta z=10$. Furthermore, for $31 \leqslant \mu \leqslant 60$, direct simulations starting from the shifted input ansatz lead to a phase instability. For instance, at $\mu=32$ and 40 , the phases of the three vertices would take values of $0, \pi / 2$, and $\pi$, instead of those displayed in Fig. 6. Actually, this instability is caused by the fact that the input is far from the shape of the stable mode, giving

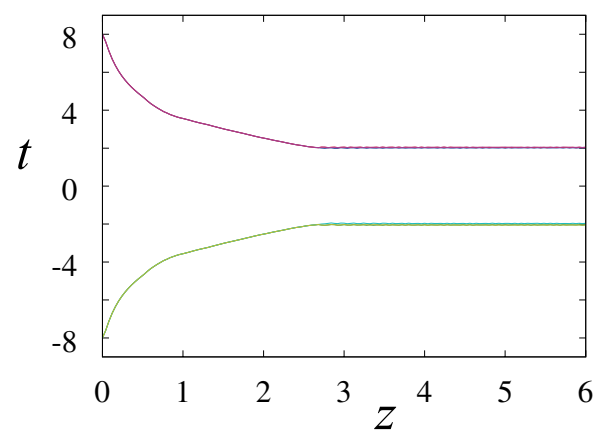

FIG. 12. (Color online) Trajectories of the motion of colliding triangular vortices rotated by angle $\pi / 3$ relative to each other, for $\mu=250$ and velocities $\pm k_{0}= \pm 11$. 


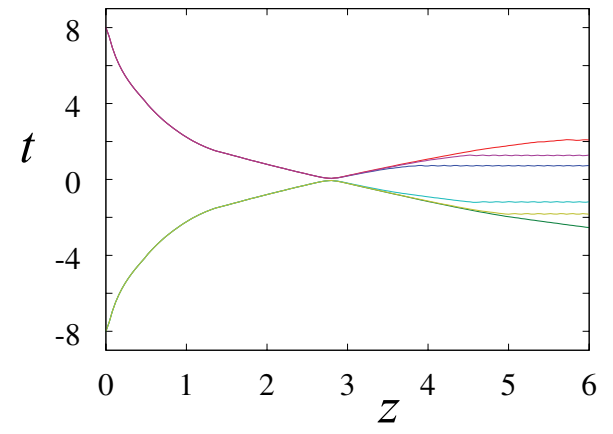

FIG. 13. (Color online) The collision of two triangular vortices at $\mu=250$ and $k_{0}=12$. Trajectories of individual pulses forming the vortices are displayed.

rise to several temporal peaks in each core. If the initial data are "cleaned up" by nullifying the field outside of the main temporal pulse, the simulations converge to stable compact triangular vortices. The amplitude of stable compact triangular vortices evolves slowly and almost linearly versus the effective propagation constant $\mu+\delta \mu$ : the computed values of the latter range from $\simeq 22-78$, and then the amplitude goes from 40.3 to 41.2 .

\section{COLLISIONS BETWEEN MOVING VORTEX SOLITONS}

The availability of stable solutions for the vortex spatiotemporal solitons and the obvious Galilean invariance of Eq. (1) suggest study of the collisions between moving vortices. In particular, it is interesting to simulate collisions between the stable triangular modes shown in Fig. 6 , rotated by angle $\pi / 3$ relative to each other, to test a possibility of their fusion into a full hexagonal vortex of the type displayed in Fig. 9. This was done by taking a pair of the triangles separated by a relatively large temporal interval, $\Delta t=16$, for values of $\mu>182$, at which the triangular vortices are stable by themselves, as shown above. They were set in motion, multiplying them by $\exp \left( \pm i k_{0} t\right)$, which obviously lends the solitons velocities $\pm k_{0}$. (In terms of the optical waveguides, these are shifts of the inverse velocities.)

In fact, the fusion of colliding triangles into a hexagon was never observed. Instead, slowly moving triangles demonstrate a long-range repulsion and stop at finite distance (but do not bounce back), as shown in Fig. 12. At intermediate velocities, the colliding triangular vortices do bounce back, and eventually they get destroyed by the longitudinal instability (splitting into uncorrelated temporal pulses in different cores), as shown in Fig. 13. At high velocities, the solitons, quite naturally, pass through each other, loosing some kinetic energy. There is a sharp threshold between the rebound regime and the passage. Just above this threshold, the passing vortices get destroyed by the longitudinal instability shortly after the collision. Domains corresponding to different outcomes of the collisions in the $\left(\mu, k_{0}\right)$ plane are shown in Fig. 14.

While the collisions seem elastic with the increase of the velocity, it was not possible to conclude if the vortices remain

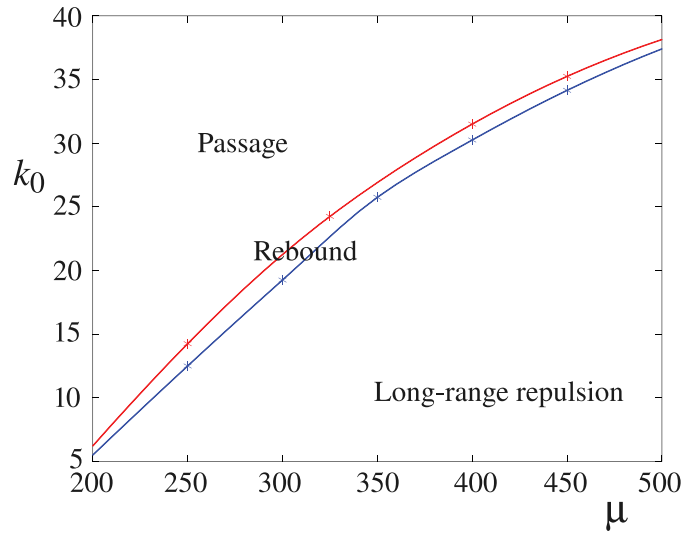

FIG. 14. (Color online) Regions of different outcomes of collisions between two mutually symmetric triangular vortices, rotated by angle $\pi / 3$.

stable indefinitely long after such quasielastic collisions. Indeed, since the numerical box has a finite length and periodic boundary conditions in $t$ are used, the moving vortices undergo repeated collisions, loosing some velocity each time. Eventually, they would be destabilized by a collision occurring at a lower speed.

\section{CONCLUSION}

We have introduced a system of parallel waveguides with the linear coupling between nearest neighbors, based on the hexagonal lattice in the transverse plane. Each guiding core features the cubic self-attractive nonlinearity. The system can find straightforward realizations in nonlinear optics and in BEC trapped in the corresponding optical lattice. Systematic simulations, starting with a natural input ansatz for vortical hexagons, reveal three distinct species of stable semidiscrete spatiotemporal complexes, which are discrete in the transverse plane and continuous in the longitudinal direction. These are triangular modes with vorticity $S=1$ and hexagonal ones with $S=2$, both built with an empty core at the center, and compact triangles carrying $S=1$, without the central empty core. Collisions between stable triangular vortices were also studied by means of simulations, demonstrating the stoppage of the slowly moving vortex solitons, destabilizing rebounds, and quasielastic passage, depending on the collision velocity.

More complex structure of the arrayed waveguides can be considered as a generalization of this work (in particular, quasiperiodic lattices). It may also be interesting to study vortex complexes in two-component models of the same type.

\section{ACKNOWLEDGMENTS}

This work was supported, in part, by a grant from the High Council for Scientific and Technological Cooperation between France and Israel. The work of D.M. at Laboratoire de Photonique d'Angers has been supported by a Grant from the Région Pays de Loire, France. 
[1] F. Lederer, G. I. Stegeman, D. N. Christodoulides, G. Assanto, M. Segev, and Y. Silberberg, Phys. Rep. 463, 1 (2008); S. Flach and A. V. Gorbach, ibid. 467, 1 (2008); Y. V. Kartashov, V. A. Vysloukh, and L. Torner, in Prog. Opt. 52, 63 (2009) (E. Wolf, editor: North Holland, Amsterdam, 2009); Eur. Phys. J. Special Topics 173, 87 (2009).

[2] Y. V. Kartashov, B. A. Malomed, and L. Torner, Rev. Mod. Phys. 83, 247 (2011).

[3] P. G. Kevrekidis, K. Ø. Rasmussen, and A. R. Bishop, Int. J. Mod. Phys. B 15, 2833 (2001).

[4] D. N. Christodoulides and R. I. Joseph, Opt. Lett. 13, 794 (1988).

[5] A. Trombettoni and A. Smerzi, Phys. Rev. Lett. 86, 2353 (2001); G. L. Alfimov, P. G. Kevrekidis, V. V. Konotop, and M. Salerno, Phys. Rev. E 66, 046608 (2002); R. Carretero-González and K. Promislow, Phys. Rev. A 66, 033610 (2002); F. S. Cataliotti, S. Burger, C. Fort, P. Maddaloni, F. Minardi, A. Trombettoni, A. Smerzi, and M. Inguscio, Science 293, 843 (2001); M. Greiner, O. Mandel, T. Esslinger, T. W. Hänsch, and I. Bloch, Nature 415, 39 (2002); N. K. Efremidis and D. N. Christodoulides, Phys. Rev. A 67, 063608 (2003).

[6] M. A. Porter, R. Carretero-González, P. G. Kevrekidis, and B. A. Malomed, Chaos 15, 015115 (2005).

[7] J. E. Heebner and R. W. Boyd, J. Mod. Opt. 49, 2629 (2002); P. Chak, J. E. Sipe, and S. Pereira, Opt. Lett. 28, 1966 (2003); J. J. Baumberg, P. G. Savvidis, R. M. Stevenson, A. I. Tartakovskii, M. S. Skolnick, D. M. Whittaker, and J. S. Roberts, Phys. Rev. B 62, R16247 (2000); P. G. Savvidis and P. G. Lagoudakis, Semicond. Sci. Technol. 18, S311 (2003).

[8] H. S. Eisenberg, Y. Silberberg, R. Morandotti, A. R. Boyd, and J. S. Aitchison, Phys. Rev. Lett. 81, 3383 (1998).

[9] T. Pertsch, U. Peschel, J. Kobelke, K. Schuster, H. Bartelt, S. Nolte, A. Tünnermann, and F. Lederer, Phys. Rev. Lett. 93, 053901 (2004).

[10] N. K. Efremidis, S. Sears, D. N. Christodoulides, J. W. Fleischer, and M. Segev, Phys. Rev. E 66, 046602 (2002); N. K. Efremidis, J. Hudock, D. N. Christodoulides, J. W. Fleischer, O. Cohen, and M. Segev, Phys. Rev. Lett. 91, 213906 (2003); J. W. Fleischer, G. Bartal, O. Cohen, T. Schwartz, O. Manela, B. Freedman, M. Segev, H. Buljan, and N. K. Efremidis, Opt. Express 13, 1780 (2005).

[11] J. W. Fleischer, M. Segev, N. K. Efremidis, and D. N. Christodoulides, Nature 422, 147 (2003); J. W. Fleischer, G. Bartal, O. Cohen, O. Manela, M. Segev, J. Hudock, and D. N. Christodoulides, Phys. Rev. Lett. 92, 123904 (2004).

[12] D. N. Neshev, T. J. Alexander, E. A. Ostrovskaya, Y. S. Kivshar, H. Martin, I. Makasyuk, and Z. Chen, Phys. Rev. Lett. 92, 123903 (2004); J. W. Fleischer, G. Bartal, O. Cohen, O. Manela, M. Segev, J. Hudock, and D. N. Christodoulides, ibid. 92, 123904 (2004).

[13] B. A. Malomed and P. G. Kevrekidis, Phys. Rev. E 64, 026601 (2001).

[14] A. Szameit, J. Burghoff, T. Pertsch, S. Nolte, A. T. Ünnermann, and F. Lederer, Opt. Express 14, 6055 (2006); A. Szameit, T. Pertsch, F. Dreisow, S. Nolte, A. Tünnermann, U. Peschel, and F. Lederer, Phys. Rev. A 75, 053814 (2007).

[15] T. Pertsch, U. Peschel, F. Lederer, J. Burghoff, M. Will, S. Nolte, and A. Tünnermann, Opt. Lett. 29, 468 (2004); A. Szameit, D. Blömer, J. Burghoff, T. Schreiber, T. Pertsch, S. Nolte,
A. Tünnermann, and F. Lederer, Opt. Express 13, 10552 (2005).

[16] P. G. Kevrekidis, B. A. Malomed, Z. Chen, and D. J. Frantzeskakis, Phys. Rev. E 70, 056612 (2004).

[17] H. Sakaguchi and B. A. Malomed, Europhys. Lett. 72, 698 (2005); R. Driben and B. A. Malomed, Eur. Phys. J. D 50, 317 (2008).

[18] J. Yang, I. Makasyuk, P. G. Kevrekidis, H. Martin, B. A. Malomed, D. J. Frantzeskakis, and Z. Chen, Phys. Rev. Lett. 94, 113902 (2005).

[19] P. G. Kevrekidis, B. A. Malomed, and Y. B. Gaididei, Phys. Rev. E 66, 016609 (2002); C. R. Rosberg, D. N. Neshev, A. A. Sukhorukov, W. Królikowski, and Y. S. Kivshar, Opt. Lett. 32, 397 (2007); T. J. Alexander, A. S. Desyatnikov, and Y. S. Kivshar, ibid. 32, 1293 (2007); B. Terhalle, T. Richter, A. S. Desyatnikov, D. N. Neshev, W. Królikowski, F. Kaiser, C. Denz, and Y. S. Kivshar, Phys. Rev. Lett. 101, 013903 (2008); K. J. H. Law, P. G. Kevrekidis, V. Koukouloyannis, I. Kourakis, D. J. Frantzeskakis, and A. R. Bishop, Phys. Rev. E 78, 066610 (2008); K. J. H. Law, H. Susanto, and P. G. Kevrekidis, Phys. Rev. A 78, 033802 (2008); K. J. H. Law, A. Saxena, P. G. Kevrekidis, and A. R. Bishop, ibid. 79, 053818 (2009).

[20] B. Terhalle et al., Phys. Rev. A 79, 043821 (2009).

[21] N. C. Panoiu, R. M. Osgood, and B. A. Malomed, Opt. Lett. 31, 1097 (2006); N. C. Panoiu, B. A. Malomed, and R. M. Osgood, Phys. Rev. A 78, 013801 (2008).

[22] P. Xie, Z.-Q. Zhang, and X. Zhang, Phys. Rev. E 67, 026607 (2003); A. Ferrando, M. Zacarés, P. F. de Cordoba, D. Binosi, and J. A. Monsoriu, Opt. Express 11, 452 (2003); 12, 817 (2004); A. Ferrando, M. Zacarés, and M. A. García-March, Phys. Rev. Lett. 95, 043901 (2005).

[23] M. J. Ablowitz, Z. H. Musslimani, and G. Biondini, Phys. Rev. E 65, 026602 (2002).

[24] I. E. Papacharalampous, P. G. Kevrekidis, B. A. Malomed, and D. J. Frantzeskakis, Phys. Rev. E 68, 046604 (2003).

[25] J. Meier, G. I. Stegeman, Y. Silberberg, R. Morandotti, and J. S. Aitchison, Phys. Rev. Lett. 93, 093903 (2004); J. Meier, G. I. Stegeman, D. N. Christodoulides, R. Morandotti, M. Sorel, H. Yang, G. Salamo, J. S. Aitchison, and Y. Silberberg, Opt. Express 13, 1797 (2005); Y. Linzon, Y. Sivan, B. Malomed, M. Zaezjev, R. Morandotti, and S. Bar-Ad, Phys. Rev. Lett. 97, 193801 (2006).

[26] D. Cheskis, S. Bar-Ad, R. Morandotti, J. S. Aitchison, H. S. Eisenberg, Y. Silberberg, and D. Ross, Phys. Rev. Lett. 91, 223901 (2003).

[27] S. Minardi et al., Phys. Rev. Lett. 105, 263901 (2010).

[28] B. A. Malomed, D. Mihalache, F. Wise, and L. Torner, J. Opt. B: Quantum Semiclass. Opt. 7, R53 (2005).

[29] A. B. Aceves, C. De Angelis, G. G. Luther, and A. M. Rubenchik, Opt. Lett. 19, 1186 (1994); E. W. Laedke, K. H. Spatschek, S. K. Turitsyn, and V. K. Mezentsev, Phys. Rev. E 52, 5549 (1995); S. Darmanyan, I. Relke, and F. Lederer, ibid. 55, 7662 (1997); A. B. Aceves, M. Santagiustina, and C. De Angelis, J. Opt. Soc. Am. B 14, 1807 (1997); I. Relke, Phys. Rev. E 57, 6105 (1998); M. Stepić, L. Hadžievski, and M. M. Skorić, ibid. 65, 026604 (2002); A. V. Yulin, D. V. Skryabin, and A. G. Vladimirov, Opt. Express 14, 12347 (2006).

[30] A. B. Aceves, C. De Angelis, A. M. Rubenchik, and S. K. Turitsyn, Opt. Lett. 19, 329 (1994); A. V. Buryak and 
N. N. Akhmediev, IEEE J. Quantum Electron. 31, 682 (1995); B. B. Baizakov, B. A. Malomed, and M. Salerno, Phys. Rev. A 70, 053613 (2004); D. Mihalache, D. Mazilu, F. Lederer, Y. V. Kartashov, L.-C. Crasovan, and L. Torner, Phys. Rev. E 70, 055603 (2004).

[31] C. J. Benton, A. V. Gorbach, and D. V. Skryabin, Phys. Rev. A 78, 033818 (2008).

[32] A. B. Aceves, G. G. Luther, C. De Angelis, A. M. Rubenchik, and S. K. Turitsyn, Phys. Rev. Lett. 75, 73 (1995).

[33] A. B. Aceves, C. De Angelis, T. Peschel, R. Muschall, F. Lederer, S. Trillo, and S. Wabnitz, Phys. Rev. E 53, 1172 (1996); A. B. Aceves and M. Santagiustina, Phys. Rev. E 56, 1113 (1997).

[34] D. Mihalache, D. Mazilu, F. Lederer, and Y. S. Kivshar, Opt. Express 15, 589 (2007); D. Mihalache, D. Mazilu, Y. S. Kivshar, and F. Lederer, ibid. 15, 10718 (2007); D. Mihalache, D. Mazilu, F. Lederer, and Y. S. Kivshar, Opt. Lett. 32, 3173 (2007); Phys. Rev. A 79, 013811 (2009); Opt. Commun. 282, 3000 (2009).

[35] D. Mihalache, D. Mazilu, F. Lederer, and Y. S. Kivshar, Opt. Lett. 32, 2091 (2007).
[36] Z. Y. Xu, Y. V. Kartashov, L. C. Crasovan, D. Mihalache, and L. Torner, Phys. Rev. E 70, 066618 (2004).

[37] H. Leblond, B. A. Malomed, and D. Mihalache, Phys. Rev. A 77, 063804 (2008).

[38] H. Leblond, B. A. Malomed, and D. Mihalache, Phys. Rev. E 76, 026604 (2007); T. Mayteevarunyoo, B. A. Malomed, B. B. Baizakov, and M. Salerno, Physica D 238, 1439 (2009).

[39] H. Leblond, B. A. Malomed, and D. Mihalache, Phys. Rev. A 79, 033841 (2009).

[40] A. Trombettoni and A. Smerzi, Phys. Rev. Lett. 86, 2353 (2001); F. Kh. Abdullaev, B. B. Baizakov, S. A. Darmanyan, V. V. Konotop, and M. Salerno, Phys. Rev. A 64, 043606 (2001); G. L. Alfimov, P. G. Kevrekidis, V. V. Konotop, and M. Salerno, Phys. Rev. E 66, 046608 (2002); R. CarreteroGonzález and K. Promislow, Phys. Rev. A 66, 033610 (2002); N. K. Efremidis and D. N. Christodoulides, ibid. 67, 063608 (2003).

[41] K. Hayata and M. Koshiba, Phys. Rev. Lett. 71, 3275 (1993). 